\title{
A survey on the provision of implants in the NHS Dental service
}

\author{
The provision of dental implants in the National Health Service Hospital Dental Services - a national questionnaire \\ by C. J. Butterworth, A. M. Baxter, M. J. Shaw and G. Bradnock Br Dent J 2001; 190:93-96
}

\begin{abstract}
Objective
To assess the activity of consultants in restorative dentistry in the United Kingdom in the provision of osseointegrated dental implants within the National Health Service Hospital service and to evaluate their attitudes concerning the relevant medical and oral factors considered in patient selection for implant treatment.
\end{abstract}

\section{Design/Setting}

Anonymous postal questionnaire in the United Kingdom.

\section{Subjects}

Consultants in restorative dentistry.

\section{Results}

Out of the sample of 145,109 consultants $(75 \%)$ completed the questionnaire in 1999.54 of the 109 consultants (49.5\%) are involved in the provision of osseointegrated implant treatment, treating an average of 29 cases/year (range 2-150). However, over one third of the respondents treated 10 or less cases/year. $89 \%$ worked with oral surgeons as an implant team. $68 \%$ used Branemark (Nobel Biocare) implants as their main system. The majority of consultants felt that smoking, psychoses and previous irradiation were the most important medical factors that contraindicated implant retained restorations whilst untreated periodontitis, poor oral hygiene and uncontrolled caries were the most important oral contra-indications. Many centres were experiencing significant problems with the funding of implant treatment with one centre receiving no funding. The implications for patient care and specialist training are discussed.

\section{Conclusions}

There is a marked variation in the number of patients treated with endosseous dental implants within the United Kingdom National Health Service hospitals. Many consultants treat 10 or fewer patients each year. In the main, there is agreement about the factors that contra-indicate implant treatment; these are in line with national guidelines.

\section{In Brief}

This article:

- Illustrates the types of patients receiving implants in the hospital dental service.

- Examines attitudes of restorative specialists to treatment planning patients for dental implants.

- Reveals problems with funding for NHS funded implant treatment.

\begin{abstract}
Comment
$\mathrm{T}$ his survey assesses implant treatment by consultants in restorative dentistry and relates some of the findings to published guidelines. The main areas covered by the survey were: the special interest of the consultant and whether he or she undertook implant treatment, the workload generated by implant treatment, whether the respondent worked in a team, medical and oral factors influencing the selection of patients for implant treatment, and the patient groups treated.

About half the respondents were involved in implant treatment but only a few treated more than 30 patients a year. One respondent purportedly treated 150 cases per year which skewed the quoted average of 29 . Not surprisingly most of the respondents indicated prosthodontics as their mono-specialty
\end{abstract}

interest. Most consultants worked in a team with an oral surgeon reflecting the demanding and complex treatment often required for these groups of patients. This is in line with the guidelines published by the Royal College of Surgeons in 1997. The authors also discuss the guideline requirement that provider units should treat sufficient numbers of patients annually to maintain expertise in this demanding area, but ask what level of activity would be appropriate?

Denture intolerance seems to be the most common problem treated with implants but only three consultants considered that management of post-cancer patients reflected the largest proportion of their case mix. Data reflected the lack of a restorative dentistry treatment service in oncology centres managing head and neck cancer in the UK despite the Calman Report emphasis on the essential improvement indicated involving quality of life issues.

The results regarding the relevance of the medical and oral factors reflected generally the published literature.

One area not covered by the questionnaire with regard to workload is maintenance which will increasingly absorb time and funding, and will inevitably limit capacity for new treatment. One of the most frequent comments was with regard to funding difficulties and this will surely be compounded by the need for ongoing maintenance of these patients.

\section{Robert A. Howell}

Consultant in Restorative Dentistry Liverpool University Dental Hospital 